\title{
Mutual antenna coupling influence on the channel correlation matrix for linear antenna arrays
}

\author{
Tatiana K. Artemova, Aleksey S. Gvozdarev, Konstantin S. Artemov
}

Department of Infocommunications and Radiophysics, P.G. Demidov Yaroslavl State University, Yaroslavl, Russia

\begin{tabular}{l}
\hline \hline Article Info \\
\hline Article history: \\
Received Apr 4, 2020 \\
Revised Oct 3, 2020 \\
Accepted Feb 28, 2021 \\
\hline
\end{tabular}

Keywords:

Antenna

Channel correlation matrix

Impedances

Matrix structure

Mutual coupling

\begin{abstract}
The paper presents the results of the research of electromagnetic mutual coupling impact on the structure of the correlation matrices in multiantenna communication systems. Classical correlation structures employed in most of the up-to-date communication systems descriptions and designs usually assume unit autocorrelation and exponentially decreasing cross-correlation of antenna elements in the receiving/transmitting array. At the same time numerous studies had shown that these assumptions may not hold under certain conditions. The performed research relates the correlation effects with the imbalances of the array impedance matrix terms and studies the impact of antenna elements mutual electromagnetic interaction upon the diagonal (autocorrelation) and off-diagonal (cross-correlation) terms of correlation matrix, depending of the geometry of the array: number of elements and their spatial separation. To exemplify quantitative results the analysis was carried out for the 5G NR \#78 band, being one of the most wideband subchannels in Under- $6 \mathrm{GHz}$ regime for $5 \mathrm{G}$ systems. The obtained results also justified the applicability of the banded correlation matrix model for wireless communications.
\end{abstract}

This is an open access article under the $\underline{C C B Y-S A}$ license.

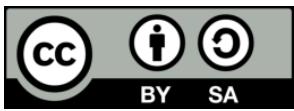

\section{Corresponding Author:}

Aleksey S. Gvozdarev

Department of Infocommunications and Radiophysics

P.G. Demidov Yaroslavl State University

Sovietskaya str. 14, 150000, Yaroslavl, Russia

Email: a.gvozdarev@ieee.org

\section{INTRODUCTION}

Communication systems' synthesis and real time adaption to the changing conditions $[1,2]$ necessitates the forecast of the communication system statistical properties [3,4]. The estimation of the potential error probability, link outage probability, channel capacity and spatial diversity gain for the case of multiple input multiple output (MIMO) systems is based on the channel matrix, which, in turn, determines the channel correlation matrix [5]. Many of these characteristics of the communication system are calculated from a set of eigenvalues of the correlation matrix. Effective techniques of dataflow distribution in MIMO systems are based on spectral decomposition of the correlation matrix. Thus, the more accurately the correlation matrix is predicted, the more accurate the estimate for the quantities of interest [5].

Usually it is assumed that the correlation matrix is exponential, i.e. its elements decrease with the spacing between the antenna elements according to the exponential or quadratic-exponential law $[3,5,6]$, with the main parameter of the correlation matrix is a one-step correlation coefficient. Physically, these two factors-the exponential law of decrease of the matrix elements and the value of the one-step correlation coefficient determine basic properties of the communication system.

In the works of Abdi and Kaveh [7-9] analytical expressions for correlation matrix elements for different channel models and various practical situations are obtained. Although being widely used in 
scientific literature, their model does not take into account the mutual coupling of elements in the antenna arrays at the base station and at the user terminal. However, as shown in a number of works, the consideration of mutual coupling affects the forecast characteristics of the communication system [10-13], thus, it must be taken into account when assuming a model of the channel correlation matrix.

Whereas for small MIMO systems it is quite easy to provide decoupling between the ports of the antenna system, it is difficult for Massive MIMO. Although a number of possible solutions for such a case are described in [14], from practical perspectives it is valuable to have an ability to estimate the significance of mutual coupling between the elements separated by some distance, hence possibly ignoring it, thus reducing the complexity of synthesized isolation networks, especially if a dense layout is planned. This can be attained by exploiting the knowledge about the structure of the system correlation matrix.

In this paper, we analyze the structure of the correlation matrix for a MIMO system equipped with dipole linear antenna arrays corresponding to the worst case in terms of mutual coupling. To reach the goal a 3D full-wave electromagnetic simulation was performed in order to analyze the structure of the antenna array mutual impedance matrix, being the key element of the correlation matrix. As the result of the simulation specific changes of the correlation matrix structure considering mutual influence are demonstrated. The analyzed effects justify the application of the proposed bordering procedure, which can possibly yield the increase in communication link quality and reduce the complexity of the of decoupling procedure.

\section{PROPOSED METHOD}

\subsection{Antenna array elements mutual coupling model}

Let us assume that the antenna array consists of $N$ identical elements forming certain geometry, for example, linear, with a constant distance $d$ between the elements. The impinging electromagnetic wave of the received signal forms a current distribution in the conductive parts or on the equivalent aperture of the $i$ th antenna element, which can be characterized by the complex envelope of the signal $I_{i}(i=1, \ldots, N)$ and the vector of the antenna array input signals $-\vec{I}=\left\{I_{i}\right\}_{i=1}^{N}$ [15]. Then, in accordance with the classical approach $[15,16]$ for each $j$-th element voltage complex envelope is defines as $U_{j}=\sum_{i=1}^{N} Z_{i j} I_{i}$. Diagonal elements $Z_{j j}$ of the $N \times N$ square impedance matrix $\mathbf{Z}$ characterize the transformation of the impinging electromagnetic field into the output voltage of this particular $j$-th element. Off-diagonal elements $Z_{j i}(i \neq j)$ are mutual impedances that characterize the effects of neighboring antenna elements. Thus, the signal vector at the output of the receiving antenna array has the form:

$$
\vec{U}=\mathbf{Z} \vec{I}
$$

Although in the most general case the expressions for mutual impedances $[17,18]$ do not allow predicting their specific values in advance, the physics of the phenomena behind the mutual coupling of the elements allows us to make a number of statements about the properties of the impedance matrix.

- First, the mutual coupling is due to multiple re-reflection of the incident signal between the elements of the antenna array, so the elements that are under different electromagnetic conditions are characterized by different self- $Z_{j j}$ and mutual resistances $Z_{j i}(i \neq j)$. So, there will be different self-impedances of elements located at the edge and in the middle of the antenna array. Similarly, the mutual coupling of neighboring elements will differ depending on whether this pair is located at the edge or in the middle of the array. This does not lead to the fact that the values of self-impedances decrease to the endings, and mutual coupling of neighboring elements by itself cannot be a measure of the electromagnetic interaction of antenna elements and a measure of the degree of correlation of signals received by different elements.

- Second, in the case of a symmetric structure of the antenna array, the impedance matrix will be Hermitian, which greatly facilitates the calculations. Moreover, $N$-element antenna array will be characterized only by $(\mathrm{N}+1)^{2} / 4$ unique values of the impedance matrix. For three-element antenna array it is 4 of 9 elements and for five-element- 9 of 25 .

- Third, since from the point of view of electrodynamics, the reflection conditions depend on the wavelength and the distance between the antenna elements, then for specific antenna elements and a specific array geometry, the elements of the impedance matrix will be functions of frequency and interelement spacing [19-21].

\subsection{Classical correlation matrix models}

Modern correlation matrix models used to analyse communication systems usually have the following structure $[5,6]$ : 


$$
\Sigma_{i, j}=r^{|i-j|}
$$

where the correlation between $i$-th and $j$-th antenna elements is assumed to depend only on the distance between the antenna elements, and is decreasing according to the exponential law, where $r$ is the one-step correlation coefficient characterizing the correlation between adjacent elements.

For communication systems the Abdi-Kaveh model is often used [7-9], which was obtained for spatial-temporal correlation of signals received by different elements of the receiving antenna array. The parameters, that characterise it are the geometry of the system, including the direction and velocity of the mobile user terminal, the number of elements in antenna arrays, the electrical distance between elements in the array (i.e., the distance normalised to the wavelength), the size of the area, where scatterers creating the conditions for multipath propagation of the signal from the base station to the user are located and the orientation of antennas of both stations. However, the authors assumed that the mutual influence of the elements can be minimized by the proper choice of the array step, so they left the mutual coupling out of consideration.

To illustrate how mutual coupling affects the correlation matrix of the system, a variant of the AbdiKaveh-Clark temporal correlation model [22] will be used in the paper. This is a model describing the Rayleigh fading channel, with the base station having a single antenna and the antenna array elements on the user side being omnidirectional. The scatterers can be arbitrarily positioned near the user, i.e. the angle directions form the base station to scatterers are described by a uniform probability distribution with zero expectation. Under these conditions the elements of the correlation matrix can be represented as [7, p. 558]

$$
\Sigma_{i, j}=J_{0}\left(\frac{2 \pi}{\lambda}|i-j| d\right)
$$

where $J_{0}(\cdot)$-zero order Bessel function of the first kind.

\subsection{Proposed correlation model modification considering antenna element mutual coupling}

Assuming the vectorised form of the receiving antenna array output (1), the signal correlation matrix is defined as;

$$
\boldsymbol{\Sigma}_{\text {mod }}=E\left[\vec{U} \vec{U}^{H}\right]=E\left[\mathbf{Z} \vec{I}(\mathbf{Z} \vec{I})^{H}\right]=E\left[\mathbf{Z} \overrightarrow{I I}^{H} \mathbf{Z}^{H}\right]=\mathbf{Z} \mathbf{\Sigma} \mathbf{Z}^{H}
$$

and is related to the correlation matrix $\Sigma$ defined by (3), where $E[\cdot]$ is the averaging operator, and $(\cdot)^{H}$ means Hermitian conjugate. Thus, the correlation matrix $\boldsymbol{\Sigma}_{\text {mod }}$ accounts for the inter-element mutual coupling since it is the result of a transformation of matrix $\mathbf{\Sigma}$ based on the mutual impedance matrix $\mathbf{Z}$. Another important result of implementing (4) is that it assumes possible non-identity of the diagonal elements, which is usually assumed in (2), (3) and other classical models, hence it gives rise to a wide variety of balancing scheme synthesis [23-28].

\section{RESEARCH METHOD}

The study was carried out on the example of linear antenna arrays of electrically shortened dipole antennas designed to work in \# 78 New Radio channels [29], i.e. at a central frequency of 3.550 GHz. The number of elements in the arrays varied from 1 to 13 with increments of 2 . For a more detailed description of the effects, the results for three values of the distance between elements normalized to the wavelength are given: 0.3 ; 0.5 and 0.7 wavelengths, which correspond to the compacted, standard and somewhat sparse placement of the array elements. The antennas had sufficient bandwidth and were made of well-conducting wire with a radius of $0.4 \mathrm{~mm}$.

Assuming the reciprocity (for transmission and reception) of the analyzed array and broadside and equal-amplitude power supply the values of electric and magnetic fields were obtained for all antenna elements and then the values of the impedance matrix entries were calculated. The analysis was performed via computational electromagnetic modeling applying finite integration technique [30] and broadband excitation.

The analysis was performed via full-wave 3D electromagnetic simulation. The setup assumed the finite integration technique procedure with open boundary conditions and solver stopping criterion of residue energy within the simulation volume $-50 \mathrm{~dB}$. The hexahedral meshing of the antenna array structure with a meshing step of not greater than 10 elements per wavelength was used. The assumed \# 78 New Radio 
channel bandwidth was increased for simulation to reach better numerical stability of the obtained results. The equally spaced antenna array was stimulated with an equal amplitude and phase broadband excitation.

\section{SIMULATION RESULTS AND DISCUSSION}

\subsection{Mutual coupling effects in linear array of dipole antennas}

To demonstrate the impact of mutual coupling numerical simulation based on the described above setup was performed. Since the simulation setup assumed dipole antennas, which are omnidirectional in the horizontal plane, the classical Jakes correlation model (3) was used as a reference. At the same time the only factor that impacts (3) is the electrical distance between the elements hence the further analysis targets the problem of its influence upon the impedance and correlation matrix elements. Nevertheless, it should be mentioned that earlier in [31] for a similar setup the effect of the array generator deformation (i.e. array bending) was studied by authors yielding results for inter-element unbalance compensation scheme synthesis in case of reconfigurable array geometry.

Figure 1 shows absolute values of the impedance matrix diagonal elements $\left|Z_{j . j}\right|$ as a function of element number $j$, i.e. self-impedances for the case of $d=\lambda / 2$. Here the plots will assume only one half of the elements in the array (for diagonal elements) since for the second half of the array (for example, for elements with numbers 8-13) the dependence can be obtained by mirroring relative to the vertical axis. For clarity, the points are connected by dotted lines. It can be seen that the self-impedance of the central element is the maximum decreasing to the edges. The relative difference is small, but with the increase of the number of elements in the antenna array, the inequality of self-impedances increases: from about $1.5 \%$ for a threeelement array to $4.5 \%$ for a 13 -element one. Thus, for large arrays, for example, used for Massive MIMO systems [4], the effect will be more significant than, for example, for widely used small $2 \times 2$ arrays MIMO systems.

Figure 2 demonstrates the impact of the interelement spacing between antennas in the array on their self-impedances $\left|Z_{j . j}\right|$ for arrays of 3, 7 and 13 elements. Hereafter, the electric distance 0.3 is marked with triangle markers, 0.5-square and 0.7-round ones. In contrast to the situation with the half-wave distance between the elements, when the self-impedances monotonically increased from the edge to the center, for arrays with steps 0.3 and 0.7 the type of dependence is oscillatory. In this case, the relative impedance inequality can be either greater or less than occurs at the standard recommended placement with a step of 0.5 wavelength. Therefore, for exploit more or less dense array elements placement, a more thorough study of the impedance matrix diagonal elements behavior is necessary.

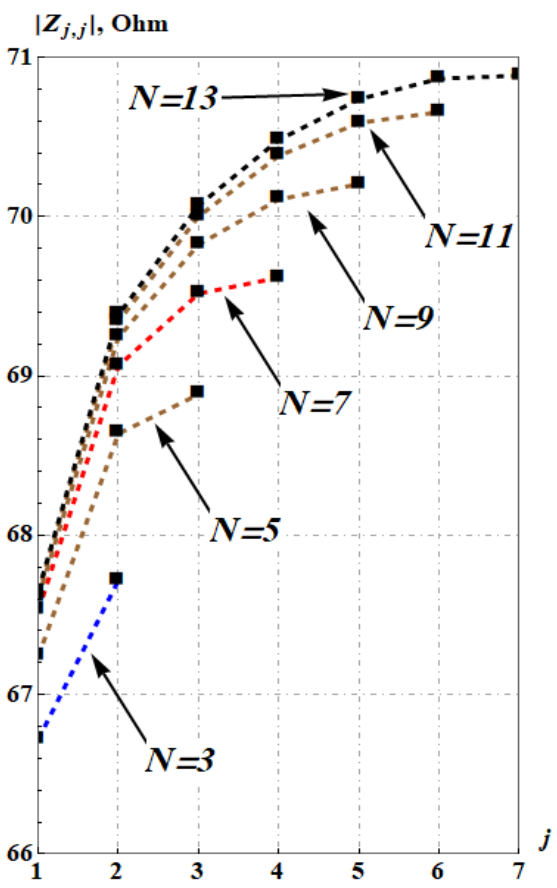

Figure 1. Absolute values of self-impedances for $d=0.5 \lambda$

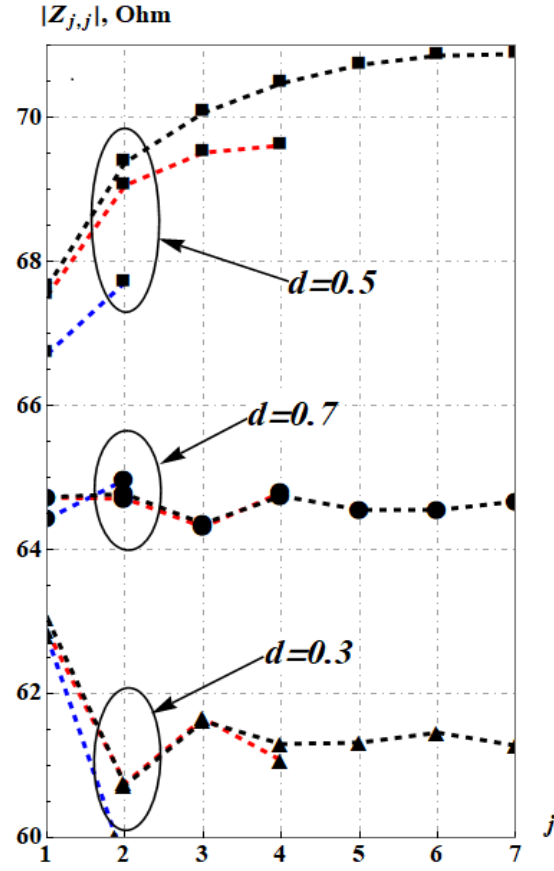

Figure 2. Absolute values of self-impedances for $d=0.3 \lambda, d=0.5 \lambda$ and $d=0.7 \lambda$ 
The analysis of off-diagonal entries of the impedance matrix for arrays of different sizes is demonstrated in Figure 3 on the example of the impedance values of the first row of the impedance matrix. It can be seen that in comparison to the self-impedance $\left|Z_{1,1}\right|$, the mutual impedances $\left|Z_{1, j}\right|$ between the first and the $j$-th elements in the array are significantly weaker, and the size of the antenna array $N$ practically does not affect the type of the dependence. However, for middle elements in the array the decline is not rapid enough to argued that only the nearest neighboring elements have a mutual coupling. Thus, for a thorough prediction of the performance of the communication system exploiting antenna arrays, it is necessary to take into account these effects.

As seen in Figure 4, the rate of decrease of the mutual coupling expressed in terms of $\left|Z_{1, j}\right|$ depends on the distance $d \cdot j$ between the elements. In denser arrays (with $d=0.3 \lambda$ ) it is stronger than in sparse one (with $d=0.7 \lambda$ ). It should be noted that the plots in Figures 3 and 4 are presented in logarithmic scale along vertical axes. Hence one can see that the substantial number of elements (but not all) exhibit linear in logarithmic scale (i.e. exponential in normal scaling) mutual coupling, which coincides with the classical exponential model. The relation suits (2) well up to the exponential base (one-step correlation coefficient), which was estimated via non-linear model fitting: for instance, $r=0.88$ in case of $d=0.5 \lambda$. At the same time closely-spaced elements and very distant element demonstrate the decrease in mutual coupling greater than exponential, which in practice should be taken into consideration.

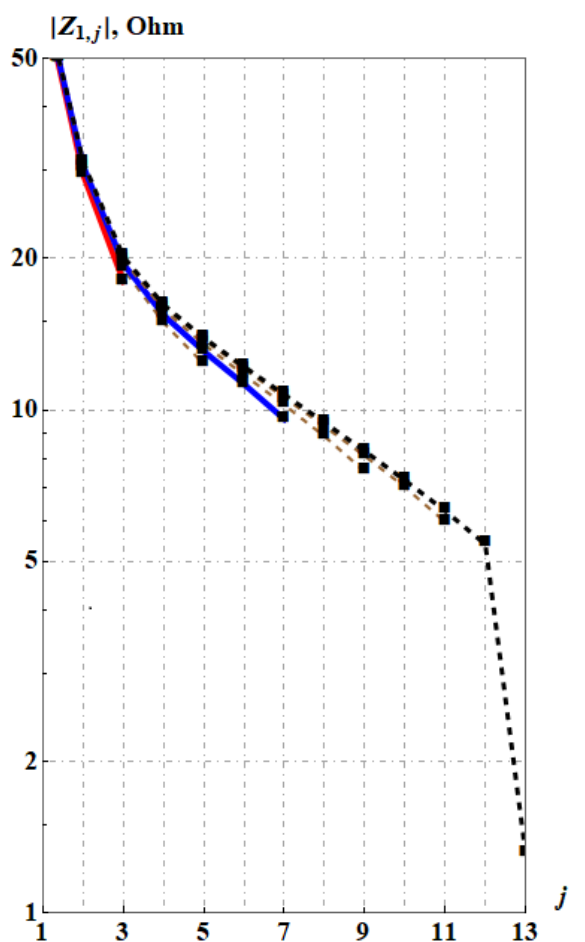

Figure 3. Absolute values of mutual impedances for different array sizes and $d=0.5 \lambda$

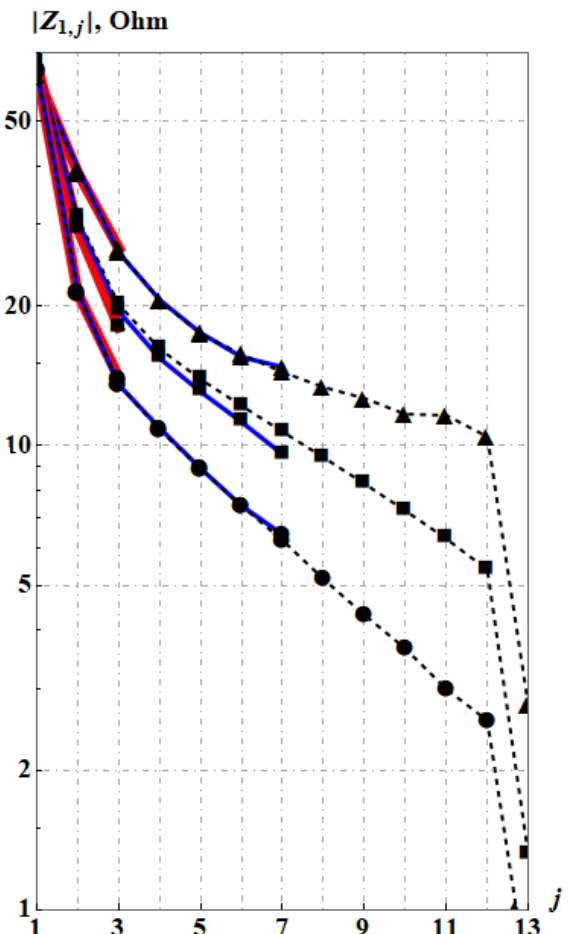

Figure 4. Absolute values of mutual impedances comparison for the cases of $d=0.3 \lambda, d=0.5 \lambda$ and $d=0.7 \lambda$

\subsection{Correlation matrices analysis}

The resultant correlation matrix $\boldsymbol{\Sigma}_{\text {mod }}$ was obtained according to the expressions (3) and (4). The effect of accounting the antenna array elements mutual coupling is presented in Figures 5 and 6 for the case of a 13-element antenna array with half-wave spacing. Figure 5 demonstrates the magnitudes of the diagonal entries of the resultant correlation matrix $\Sigma_{\text {mod }}$ (blue line with round markers) and the matrix $\Sigma$ obtained in accordance with (3) (red line with square markers). As one would expect from Figure 1, the imbalance between the values of the self-impedances of the elements in the array leads to a difference between the values of the diagonal elements of the correlation matrix compared to a conventional one in which the main diagonal equal to unity. However, the observed difference is not as significant as the difference in the decrease of off-diagonal entries, which is shown in Figure 6. 


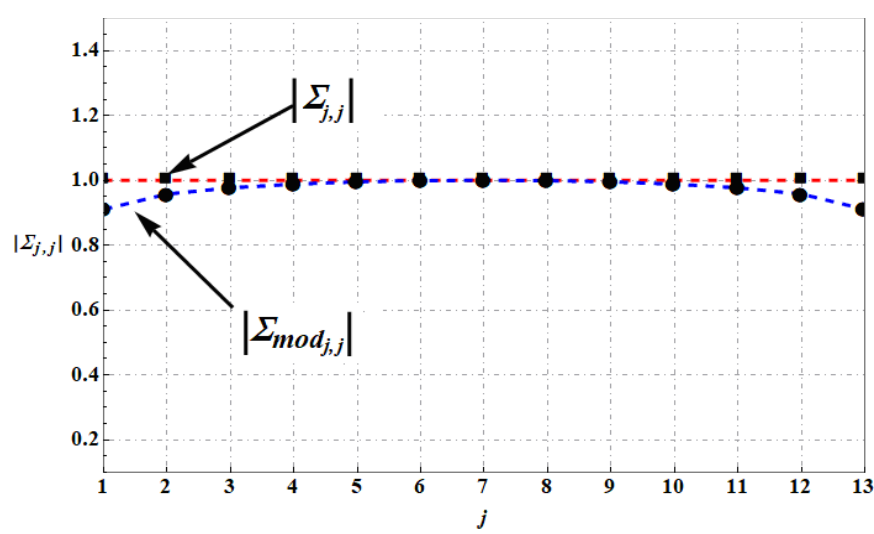

Figure 5. Comparison of correlation matrices diagonal elements' magnitudes for two models and $d=0.5 \lambda$

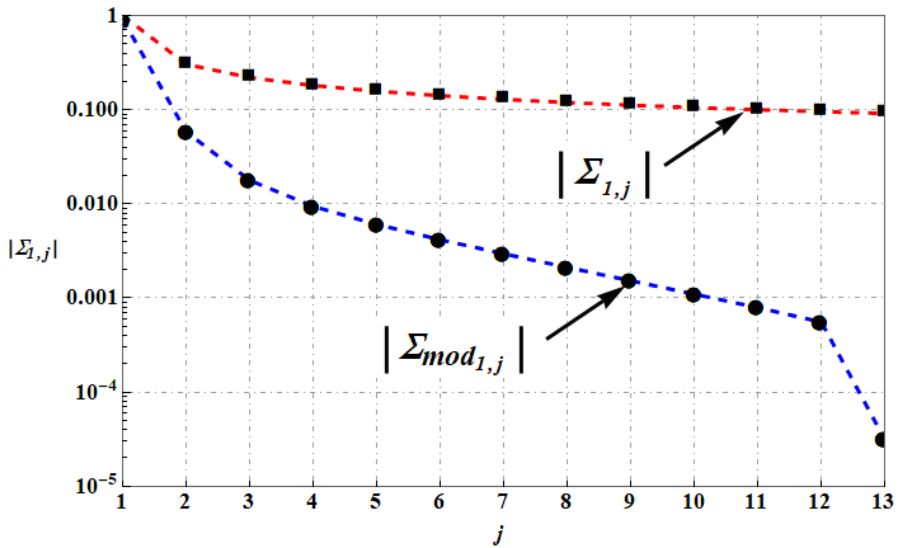

Figure 6. Comparison of correlation matrices off-diagonal elements' magnitudes for two models and

$$
d=0.5 \lambda
$$

It can be seen that compared to the classical Clark model (3) the decrease of the cross-correlation properties is much greater (for example by 2 orders of magnitude for a 10-element array) and cannot be controlled by the exponential model. Hence it can be argued whether the impact of distant elements is small enough to be excluded from further evaluations thus resulting in banded correlation matrix structures [32]. As it was demonstrated earlier in [33] this can possibly yield further improvement in system performance. For example, authors have shown (see [33]) that for a Massive MIMO system with available channel state information at both sides (transmitter- and receiver-) exploiting eigenmode transmission with waterfilling power allocation the procedure of tridiagonal bordering of the correlation matrix introduces possible MIMO channel capacity gain that reaches $7 \%$ for a $64 \times 64$ antenna array relatively to the full correlation matrix case. The electrodynamic justification of such a procedure is partially given by the stated results, see Figure 6 .

\section{CONCLUSION}

The research presents qualitative and quantitative results concerning the impact of the mutual coupling upon the diagonal (corresponding autocorrelation) and off-diagonal (corresponding crosscorrelation) terms of correlation matrix, depending on the geometry of the linear array: number of elements and their spatial separation, which reflects the cases of classical spacing and dense/sparse array layouts. Quantitative analysis demonstrated that the classical Clark correlation model is inappropriate in massive MIMO systems with mutual coupling, since autocorrelation does not constitute to unity and, hence, cannot be governed by the classical exponential model. So, in practice it is necessary either design decoupling schemes or modify correlation matrix model used for signal precoding and decoding in a way that is proposed in this work. Qualitative analysis shows the possibility to use banded correlation matrix model almost without losing the accuracy of evaluating the main characteristics of the communication system for a Massive MIMO system.

Mutual antenna coupling influence on the channel correlation matrix for linear... (Tatiana K. Artemova) 


\section{ACKNOWLEDGEMENTS}

Authors would like to acknowledge that the research was performed within P.G. Demidov Yaroslavl State University research program № ОП-2Г-06-2019.

\section{REFERENCES}

[1] M. S. R. Bashri, T. Arslan and W. Zhou, "Flexible antenna array for wearable head imaging system," 11th European Conference on Antennas and Propagation (EUCAP), pp. 172-176, 2017.

[2] Q. Jia, H. Xu, M. F. Xiong, B. Zhang and J. Duan, "Omnidirectional solid angle beam-switching flexible array antenna in millimeter wave for 5G micro base station applications," IEEE Access, vol. 7, pp. 157027-157036, 2019.

[3] M. K. Simon, M. S. Alouini, "Digital communication over fading channels: a unified approach to performance analysis," Wiley-Interscience, 2000.

[4] GTI, "Massive MIMO White Paper, V. 02, Global TD-LTE Initiative," 2018. [Online]. Availabe at: http://ydgtix.dev.ftbj.net/skin/revision/image/GTI_Massive_MIMO_White_Paper_v2.0.pdf.

[5] G. Levin, "Capacity analysis of asymptotically large MIMO channels," Ph. D. dissertation, Ottawa-Carleton Institute for Electrical and Computer Engineering, School of Information Technology and Engineering, University of Ottawa, Ottawa, Ontario, Canada, 2008.

[6] J-H. Lee, C-C. Cheng, "Spatial correlation of multiple antenna arrays in wireless communication systems," Progress In Electromagnetics Research, vol. 132, pp. 347-368, 2012.

[7] A. Abdi and M. Kaveh, "A space-time correlation model for multielement antenna systems in mobile fading channels," IEEE Journal on Selected Areas in Communications, vol. 20, no. 3, pp. 550-560, April 2002.

[8] M. O. Damen, A. Abdi and M. Kaveh, "On the effect of correlated fading on several space-time coding and detection schemes," IEEE 54th Vehicular Technology Conference. VTC Fall 2001. Proceedings (Cat. No.01CH37211), vol. 1, pp. 13-16, 2001.

[9] A. Abdi and M. Kaveh, "A versatile spatio-temporal correlation function for mobile fading channels with nonisotropic scattering," Proceedings of the Tenth IEEE Workshop on Statistical Signal and Array Processing (Cat. No.00TH8496), pp. 58-62, 2000.

[10] T. Svantesson, "The effects of mutual coupling using a linear array of thin dipoles of finite length," Ninth IEEE Signal Processing Workshop on Statistical Signal and Array Processing (Cat. No.98TH8381), pp. 232-235, 1998.

[11] Z. Qing, P. Zong and D. R. Becerra, "The analysis and compensation of the mutual coupling for the adaptive array antenna," 2010 International Conference on Microwave and Millimeter Wave Technology, pp. 403-406, 2010.

[12] D. B. Alexander, R. M. Narayanan and B. Himed, "Lower bounds for wideband direction-finding with mutual coupling," 2019 IEEE Radar Conference (RadarConf), pp. 1-6, 2019.

[13] E. Michailidis, C. Tsimenidis and G. Chester, "Mutual coupling reduction in a linear two element patch array and its effect on theoretical MIMO capacity," 2008 Loughborough Antennas and Propagation Conference, pp. 457460, 2008.

[14] X. Chen, S. Zhang and Q. Li, "A review of mutual coupling in MIMO systems," IEEE Access, vol. 6, pp. 2470624719, 2018

[15] Hui, "A new definition of mutual impedance for application in dipole receiving antenna arrays," IEEE Antennas and Wireless Propagation Letters, vol. 3, pp. 364-367, 2004.

[16] G. D. Durgin and T. S. Rappaport, "Effects of multipath angular spread on the spatial cross-correlation of received voltage envelopes," 1999 IEEE 49th Vehicular Technology Conference (Cat. No.99CH36363), vol. 2, pp. 996 $1000,1999$.

[17] H. A. Abdallah and W. Wasylkiwskyj, "An efficient numerical technique for calculating mutual coupling in antenna arrays based on the characteristics of an isolated element," 2005 IEEE Antennas and Propagation Society International Symposium, vol. 3B, pp. 43-46, 2005.

[18] M. Sierra Castaner, J. L. Masa, O. Moreno, J. M. Fernandez and D. Castellanos, "Estimation of patch array coupling model through radiated field measurements," IEEE Antennas and Propagation Society International Symposium. Digest. Held in conjunction with: USNC/CNC/URSI North American Radio Sci. Meeting (Cat. No.03CH37450), Columbus, OH, 2003, vol. 1, pp. 610-613, 2003.

[19] P. J. D. Gething, "Mutual-impedance effects for a linear aerial array," Electronics Letters, vol. 2, no. 10, pp. 387388, October 1966.

[20] D. Sinnott, "Matrix analysis of linear antenna arrays of equally spaced elements," IEEE Transactions on Antennas and Propagation, vol. 21, no. 3, pp. 385-386, May 1973.

[21] C. A. Balanis, "Antenna theory," 2nd edition, John Wiley \& Sons, 1997.

[22] W. C. Jakes Jr., "Microwave mobile communications: Multipath interference," IEEE Press, pp. 11-78, 1974.

[23] L. G. Sodin, "Frequency-independent approximate compensation of mutual coupling in a linear array antenna," IEEE Transactions on Antennas and Propagation, vol. 57, no. 8, pp. 2293-2296, Aug. 2009.

[24] L. G. Sodin, "Method of synthesizing a beam-forming device for the N-beam and N-element array antenna, for any N," IEEE Transactions on Antennas and Propagation, vol. 60, no. 4, pp. 1771-1776, April 2012.

[25] L. Sun, G. Zhang and B. Sun, "Method of synthesizing orthogonal beam-forming networks using QR decomposition," IEEE Access, vol. 7, pp. 325-331, 2019.

[26] H. J. Chaloupka and X. Wang, "On the properties of small arrays with closely spaced antenna elements," IEEE Antennas and Propagation Society Symposium, 2004., vol. 3, pp. 2699-2702, 2004. 
[27] T. Basikolo, K. Ichige and H. Arai, "A novel mutual coupling compensation method for underdetermined direction of arrival estimation in nested sparse circular arrays," IEEE Transactions on Antennas and Propagation, vol. 66, no. 2, pp. 909-917, Feb. 2018.

[28] J. Zhang, S. Yan, X. Hu and G. A. E. Vandenbosch, "Reduction of mutual coupling for wearable antennas," 2019 13th European Conference on Antennas and Propagation (EuCAP), pp. 1-2, 2019.

[29] ETSI, "NR User Equipment (UE) radio transmission and reception; Part 1: Range 1 Standalone" 3GPP TS 38.101-1 V16.4.0 release 16, 2020. Available at: https://www.etsi.org/deliver/etsi_ts/138100_138199/13810101/16.04.00_60/ts_13810101v160400p.pdf.

[30] D. B. Davidson, "Computational electromagnetics for RF and microwave engineering," 2nd edition, Cambridge University Press, 2010.

[31] T. K. Artemova and A. S. Gvozdarev, "Antenna array characteristics influence on MIMO system reliability," 2019 Systems of Signal Synchronization, Generating and Processing in Telecommunications (SYNCHROINFO), pp. 1-5, 2019.

[32] A. Gvozdarev and T. Artemova, "Banded correlation matrix model for massive MIMO systems," 2017 IEEE EastWest Design \& Test Symposium (EWDTS), pp. 1-6, 2017.

[33] A. S. Gvozdarev and T. K. Artemova, "Massive MIMO system capacity analysis in case of banded correlation matrix model application," 2020 Moscow Workshop on Electronic and Networking Technologies (MWENT), pp. 1$6,2020$.

\section{BIOGRAPHIES OF AUTHORS}
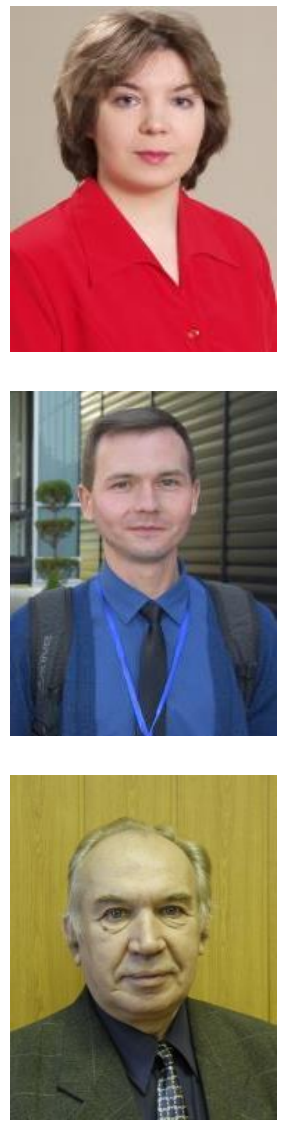

Tatiana K. Artemova received her Specialist Degree (Radiophysics and electronics) in 1994 from P.G. Demidov Yaroslavl State University, Yaroslavl, Russia and Ph.D degree (Numerical methods and simulation in physics and mathematics) in 1998 from P.G. Demidov Yaroslavl State University, Yaroslavl, Russia. She is now an Associate Professor of Infocommunications and Radiophysics at P.G. Demidov Yaroslavl State University. Her current research interests are in the field of wireless communications, antennas and applied electrodynamics.

Aleksey S. Gvozdarev received his Specialist Degree (Radiophysics and electronics) in 2007 from P.G. Demidov Yaroslavl State University, Yaroslavl, Russia and Ph.D degree (Radiophysics) in 2015 from Voronezh State University, Voronezh, Russia. He is now an Associate Professor of Infocommunications and Radiophysics at P.G. Demidov Yaroslavl State University. His current research interests are in the field of wireless communications, signal processing, wireless channels models, information theory and applied electrodynamics. He is a long-term IEEE member of such societies as: "Antennas and Propagation Society", "Signal Processing Society", "Communication Society" and "Information Theory Society".

Konstantin S. Artemov received his Specialist Degree (Semiconductor materials) in 1965 from Voronezh State University, Voronezh, Russia and Ph.D degree (Radiophysics) in 1975 from Voronezh State University, Voronezh, Russia. He is now an Associate Professor of Infocommunications and Radiophysics at P.G. Demidov Yaroslavl State University. His current research interests are in the field of wireless communications, circuitry, microwave imaging and applied electrodynamics. 